\title{
MicroRNA-873 is a Potential Serum Biomarker for the Detection of Ectopic Pregnancy
}

\author{
Qi Lu Qi Yan ${ }^{\mathrm{b}}$ Fengying Xuc Yuhong Lia Wenxia Zhao ${ }^{\mathrm{b}}$ Chunzhu Wu ${ }^{\mathrm{b}}$ \\ Yudong Wang ${ }^{\text {a }}$ Xiao Lang ${ }^{\text {a }}$
}

aDepartment of Obstetrics and Gynecology, International Peace Maternity and Child Health Hospital, Shanghai Jiao Tong University School of Medicine, Shanghai, bepartment of Obstetrics and Gynecology, Jiangwan Hospital of Shanghai Hongkou District, Shanghai, 'Department of Obstetrics and Gynecology, Tinglin Hospital of Shanghai Jinshan District, Shanghai, China

\section{Key Words \\ Ectopic pregnancy $\bullet$ Biomarker $・$ MicroRNA-873 $・$ Diagnosis}

\begin{abstract}
Background: Ectopic pregnancy (EP) refers to the implantation of the zygote outside the uterine cavity. In clinical practice, the diagnosis of EP relies on a combination of ultrasound findings and serum human chorionic gonadotrophin (hCG) measurements. However, the need for serial hCG measurements increases the risk of tubal rupture and death, underscoring the need to identify biomarkers for the early detection of EP. Methods: The serum concentrations of 21 microRNAs (miRNAs) associated with pregnancy or with known placental expression, as well as serum hCG and progesterone levels were analyzed 36 patients with viable intrauterine pregnancy (VIP), 30 patients with spontaneous abortion (SA), and 34 patients with EP using specific assay kits and reverse transcription PCR. The diagnostic performance of the different serum markers for detecting EP was analyzed by ROC curve analysis. Results: Five miRNAs were differentially expressed between the three groups, of which miR-873 and miR-223 were significantly lower in EP than in VIP and SA patients and did not change significantly according to gestational age, and miR-323 was significantly higher in EP than in VIP and SA. As a single marker, miR-873 had the highest sensitivity for detecting EP at $61.76 \%$ (at a fixed specificity of $90 \%$ ). In comparison, the combination of hCG, progesterone and miR-873 had the highest sensitivity for detecting EP at $79.41 \%$ (at a fixed specificity of $90 \%$ ). Conclusion: Although further validation in large-scale prospective studies is necessary, our results suggest that miR873 could be a valuable noninvasive and stable biomarker for the early detection of EP.
\end{abstract}

\section{Introduction}

Ectopic pregnancy (EP) is a condition in which the fertilized egg implants in a location other than the inner lining of the uterus [1]. The incidence of EP has increased markedly Q. Lu and Q. Yan contributed equally to this work. 
in the last decades from 4.5 per 1000 pregnancies in 1970 to an estimated 19.7 per 100 pregnancies in 1992, and it remains an important cause of maternal death, accounting for approximately $9-13 \%$ of pregnancy-associated deaths in developed countries and $10-30 \%$ in African developing countries [2,3]. EP is diagnosed by transvaginal ultrasonography, which detects an extrauterine gestational sac containing a yolk sac; however, additional assessment by surgical or biochemical methods is necessary in approximately $8-31 \%$ of patients [4]. The most common biochemical markers for the diagnosis of EP are serum human chorionic gonadotropin (hCG) and progesterone. Progesterone is an indicator of fetal viability and concentrations above $25 \mathrm{ng} / \mathrm{ml}$ indicate a viable intrauterine pregnancy (VIP), whereas concentrations below $5 \mathrm{ng} / \mathrm{ml}$ are non-viable [5]. For hCG, serial monitoring every $48 \mathrm{~h}$ is necessary for a positive diagnosis of EP; an increase in $\mathrm{hCG}$ of $\geq 66 \%$ in $48 \mathrm{~h}$ is associated with VIP, whereas an increase of $<50 \%$ is associated with abnormal pregnancy including EP $[6,7]$. However, these measurements cannot identify the location of the gestational sac and are associated with high false-positive and false-negative rates, in addition to putting the patient at risk of tubal rupture in between measurements [8]. Furthermore, these markers are not effective for distinguishing between EP and spontaneous abortion [9]. Therefore, there is a need to develop accurate and sensitive noninvasive tests for the diagnosis of EP.

MicroRNAs (miRNAs) are small (22 nucleotides) endogenous single-stranded RNAs that regulate gene expression by binding to the $3^{\prime}$ untranslated region of target genes, leading to translational repression or degradation of the targeted transcript [10]. MiRNAs are aberrantly expressed in many diseases, and their stability and resistance to degradation makes them useful biomarkers in cancer, autoimmune diseases, sepsis, and acute myocardial infarction among others [11]. Pregnancy-associated circulating miRNAs have been researched extensively in recent years as potential biomarkers for the diagnosis of pregnancy-associated complications, and alterations in the concentration of these miRNAs has been shown to reflect pathological states such as preeclampsia, preterm labor, and fetal growth restriction among others [12-16].

In the present study, we examined the serum concentration of different miRNAs to identify pregnancy-associated miRNAs differentially expressed in patients with EP, SA, and VIP and analyzed their diagnostic accuracy as biomarkers for the detection of EP in an emergency department (ED) setting.

\section{Materials and Methods}

Specimen collection

Patients were recruited from the International Peace Maternity and Child Health Hospital and JiangWan Hospital, Shanghai. All patients included agreed to participate in the study, and the study was approved by Ethical Board of Shanghai Jiao Tong University School of Medicine. Remnant serum samples sent to the laboratory from the ED for physician-ordered hCG testing between 2013 and 2016 were included in the analysis. All serum samples sent to the laboratory for quantitative hCG testing were retained and frozen at $-70^{\circ} \mathrm{C}$ before progesterone and miRNA testing was performed.

\section{HCG and progesterone testing}

All hCG and progesterone measurements were performed at International Peace Maternity and Child Health Hospital and JiangWan Hospital using commercially available assays. Serum hCG and serum progesterone were assayed using the Centaur Total hCG assay and the Centaur Progesterone assay, respectively.

\section{Serum Isolation and RNA extraction}

Total RNA containing miRNAs was extracted from $250 \mu \mathrm{L}$ serum using the TRIzol LS reagent (Invitrogen, Carlsbad, CA) according to the manufacturer's protocol with modifications.

\section{Primers}

All primers were synthesized and purchased from Integrated DNA Technologies (IDT, Coralville, IA), and the primers of candidate miRNAs are listed in Table 1. 


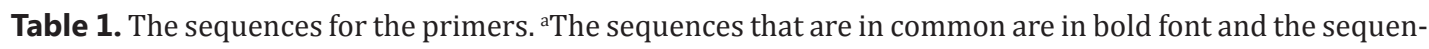
ces that are miRNA specific are in italic fonts. ${ }^{b}$ Universal Reverse primer

\begin{tabular}{|c|c|c|}
\hline Target & stem-loop Reverse primera & forward primera \\
\hline hsa-mir-196b & CTCAACTGGTGTCGTGGAGTCGGCAATTCAGTTGAGGAAGGCAG & ACACTCCAGCTGGGTCGACAGCACGACACT \\
\hline hsa-mir-30a & CTCAACTGGTGTCGTGGAGTCGGCAATTCAGTTGAGGCAGCTGC & ACACTCCAGCTGGGCTTTCAGTCGGATGTTTG \\
\hline hsa-mir-873 & CTCAACTGGTGTCGTGGAGTCGGCAATTCAGTTGAGTCCCGGGA & ACACTCCAGCTGGGGGAGACTGATGAGT \\
\hline hsa-mir-337-3p & CTCAACTGGTGTCGTGGAGTCGGCAATTCAGTTGAGGAAGAAAG & ACACTCCAGCTGGGCTCCTATATGATGC \\
\hline hsa-mir-1288 & CTCAACTGGTGTCGTGGAGTCGGCAATTCAGTTGAGTCTCCAGA & ACACTCCAGCTGGGTGGACTGCCCTGA \\
\hline hsa-mir-451 & CTCAACTGGTGTCGTGGAGTCGGCAATTCAGTTGAGAACTCAGT & ACACTCCAGCTGGGAAACCGTTACCATT \\
\hline hsa-mir-223 & CTCAACTGGTGTCGTGGAGTCGGCAATTCAGTTGAGTGGGGTAT & ACACTCCAGCTGGGTGTCAGTTTGTCAA \\
\hline hsa-mir-141 & CTCAACTGGTGTCGTGGAGTCGGCAATTCAGTTGAGCCATCTTT & ACACTCCAGCTGGGTAACACTGTCTGGTAA \\
\hline hsa-mir-151-5p & CTCAACTGGTGTCGTGGAGTCGGCAATTCAGTTGAG $A C T A G A C T$ & ACACTCCAGCTGGGTCGAGGAGCTCACAG \\
\hline hsa-mir-520a-5p & CTCAACTGGTGTCGTGGAGTCGGCAATTCAGTTGAGAGAAAGTA & ACACTCCAGCTGGGCTCCAGAGGGAAGTA \\
\hline hsa-mir-525-3p & CTCAACTGGTGTCGTGGAGTCGGCAATTCAGTTGAGCGCTCTAA & ACACTCCAGCTGGGGAAGGCGCTTCCCTT \\
\hline hsa-mir-433 & CTCAACTGGTGTCGTGGAGTCGGCAATTCAGTTGAGACACCGAG & ACACTCCAGCTGGGATCATGATGGGCTCCT \\
\hline hsa-mir-487a & CTCAACTGGTGTCGTGGAGTCGGCAATTCAGTTGAG $A A C T G G A T$ & ACACTCCAGCTGGGAATCATACAGGGACAT \\
\hline hsa-mir-218 & CTCAACTGGTGTCGTGGAGTCGGCAATTCAGTTGAGACATGGTT & ACACTCCAGCTGGGTTGTGCTTGATCTAA \\
\hline hsa-mir-204 & CTCAACTGGTGTCGTGGAGTCGGCAATTCAGTTGAG $A G G C A T A G$ & ACACTCCAGCTGGGTTCССTTTGTCATCC \\
\hline hsa-mir-516-5p & CTCAACTGGTGTCGTGGAGTCGGCAATTCAGTTGAGAAAGTGCT & ACACTCCAGCTGGGATCTGGAGGTAAGAAG \\
\hline hsa-mir-517 & CTCAACTGGTGTCGTGGAGTCGGCAATTCAGTTGAG $A C A C T C T A$ & ACACTCCAGCTGGGATCGTGCATCCCTT \\
\hline hsa-mir-518b & CTCAACTGGTGTCGTGGAGTCGGCAATTCAGTTGAG $A C C T C T A A$ & ACACTCCAGCTGGGCAAAGCGCTCСССТTT \\
\hline hsa-mir-526a & CTCAACTGGTGTCGTGGAGTCGGCAATTCAGTTGAGCAGAAAGT & ACACTCCAGCTGGGCTCTAGAGGGAAGCAC \\
\hline hsa-mir-154 & CTCAACTGGTGTCGTGGAGTCGGCAATTCAGTTGAGAATAGGTC & ACACTCCAGCTGGGAATCATACACGGTTGA \\
\hline has-mir-323-3p & CTCAACTGGTGTCGTGGAGTCGGCAATTCAGTTGAGAGAGGTCG & ACACTCCAGCTGGGCACATTACACGGTCG \\
\hline universalR ${ }^{b}$ & \multicolumn{2}{|l|}{$\begin{array}{l}\text { TGGTGTCGTGGAGTCG }\end{array}$} \\
\hline $18 \mathrm{~s}$ & CACAGTTATCCAAGTGGGAGAGG & GAAACTGCGAATGGCTCATTAAA \\
\hline
\end{tabular}

Reverse transcriptase reactions

For each reaction, $4 \mu \mathrm{L}$ RNA, $5 \mathrm{nmol} / \mathrm{L}$ of each miRNA-specific reverse stem-loop primer, $1 \times \mathrm{RT}$ buffer, $0.25 \mathrm{mM}$ each of dNTPs, $1 \mu \mathrm{L}$ MultiScribe reverse transcriptase and $1 \mu \mathrm{L}$ RNase inhibitor were added into a $10 \mu \mathrm{L}$ multiplex reverse transcription reaction (High-Capacity cDNA Archive Kit; Applied Biosystems). Reactions were incubated in a96-well plate for $30 \mathrm{~min}$ at $16^{\circ} \mathrm{C}, 30 \mathrm{~min}$ at $42^{\circ} \mathrm{C}, 5 \mathrm{~min}$ at $85^{\circ} \mathrm{C}$ and then held at $4^{\circ} \mathrm{C}$.

\section{Real-time quantitative reverse-transcription (RT) PCR analysis}

The $10 \mu \mathrm{L}$ multiplex PCR reaction included $0.5 \mu \mathrm{L}$ RT product, $1 \times$ TaqMan Universal PCR Master PCR Mix, $1 \mu \mathrm{mol} / \mathrm{L}$ of the universal reverse primer, $1 \mu \mathrm{mol} / \mathrm{L}$ each miRNA forward primer and $0.1 \mu \mathrm{mol} / \mathrm{L}$ of TaqMan probe. PCR reactions were analyzed on an Applied Biosystems 7500 Fast Real-Time PCR System. PCR conditions were $95^{\circ} \mathrm{C}$ for $10 \mathrm{~min}$, and then 40 cycles of $95^{\circ} \mathrm{C}$ for $15 \mathrm{~s}$ and $60^{\circ} \mathrm{C}$ for $1 \mathrm{~min}$. Ct values were calculated and converted into fold-change relative to that of $18 \mathrm{~s}$ RNA.

\section{Statistical analysis}

Patient age, gestational age (GA), and concentrations of serum hCG and progesterone, and miRNAs of the EP, SA, and VIP patients were compared. Because the distributions of these variables were not Gaussian, the Kruskal-Wallis test and the Dunn multiple-comparison post hoc test were used to determine statistical significance. ROC curve analysis was performed, and areas under the curve (AUCs) were calculated. Sensitivity and specificity were calculated using Pearson $\chi 2$ analysis. The cutoff point of each step was determined by fixing the sensitivity at the highest level. The ANOVA test, box plot were performed with GraphPad Prism (version 5.0; GraphPad Software), and ROC analysis was performed with SPSS (version 17.0; SPSS Inc., Chicago, IL). Statistical significance was defined as a P value $<0.05$.

\section{Results}

Serum concentrations of hCG, progesterone, miR-218, miR-223, miR-141, miR-873 and miR-323 in patients with EP, SA, and VIP

A total of 36 patients with VIP, 34 patients with EP, and 30 patients with SA were included in the analysis, and the median (range) ages were 28 (25-31.5), 30 (27-34), and 28.5 (25-33) years, respectively. There were no significant differences in age or GA between the three groups. Patient characteristics and serum values of hCG, progesterone, and specific miRNAs in patients with EP, SA, and VIP are shown in Table 2. Serum hCG and progesterone 
Fig. 1. Expression of hCG, progesterone and five miRNAs in EP, SA and VIP patients. (A-G) Box plots of measurements of hCG, progesterone, miR-218, miR-223, miR-141, miR-873 and miR-323 in patients with EP, SA, and VIP. The median, interquartile range, and the data range are shown; outliers are also indicated. $\quad * \mathrm{P}<0.5, \quad * * \mathrm{P}$ $<0.01$ and $* * * \mathrm{P}<0.001$, Dunn multiple-comparison test.

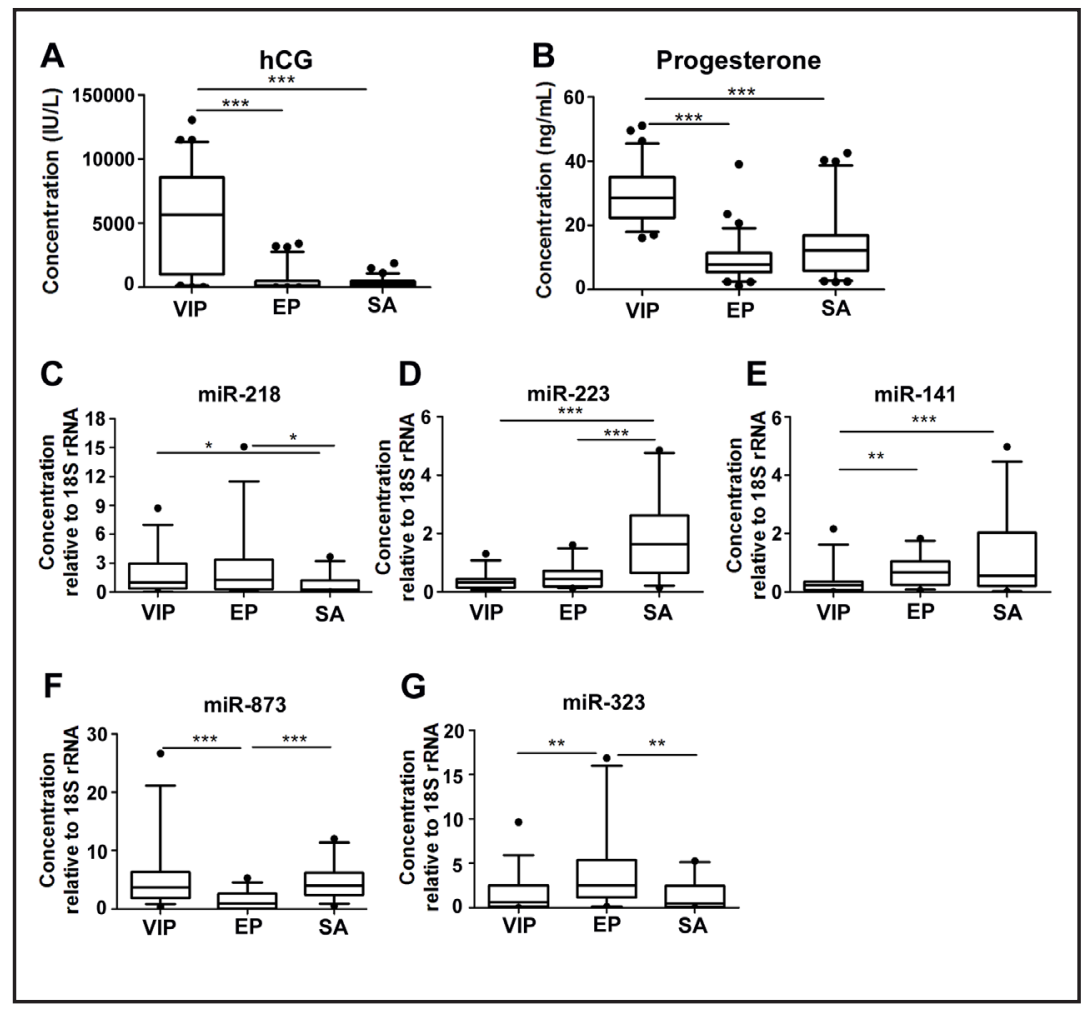

concentrations were significantly lower in patients with EP and SA than in those with VIP $(\mathrm{P}<0.01$, Fig. 1A and 1B), and lower in EP than in SA, although the difference did not reach statistical significance. A total of 21 miRNA candidates (hsa-mir-196b, hsa-mir-30a, hsamir-873, hsa-mir-337-3p, hsa-mir-1288, hsa-mir-451, hsa-mir-223, hsa-mir-141, hsamir-151-5p, hsa-mir-520a-5p, hsa-mir-525-3p, hsa-mir-433, hsa-mir-487a, hsa-mir-218, hsa-mir-204, hsa-mir-516-5p, hsa-mir-517, hsa-mir-518b, hsa-mir-526a, hsa-mir-154 and has-mir-323-3p) with a known association with pregnancy or placental specificity were analyzed to identify potential candidate miRNAs capable of distinguishing between women with EP, VIP, and SA. We identified miR-218, miR-223, miR-873, miR-141 and miR-323 as differentially expressed between the three groups. The serum concentration of miR-218 was higher in EP and VIP than in SA ( $P<0.05$ both, Fig. 1C), but did not differ significantly between EP and VIP; miR-223 was lower in VIP than in EP and SA $(\mathrm{P}<0.001$, Fig. 1D) but did not differ significantly between EP and VIP; miR-141 was higher in EP and SA than in VIP $(\mathrm{P}<0.01$ and $\mathrm{P}<0.001$, Fig. 1E) but did not differ significantly between EP and SA; miR-873 was lower in EP than in SA and VIP (P < 0.001 both, Fig. 1F); however, miR-323 was significantly higher in EP than in VIP and SA (P $<0.01$ both, Fig. 1G) (Table 2). Analysis of the correlations between the serum concentrations of hCG and progesterone and GA in the different groups showed that hCG was significantly positively correlated with GA in the VIP group (Fig. 2A), and progesterone concentration was negatively correlated with GA in the VIP group, but not in the EP or SA groups (Fig. 2B). MiR-218 expression was positively correlated with GA only in the VIP group (Fig. 2C). However, miR-223, miR-141, miR-873 and miR-323 concentrations in the EP, SA, and VIP groups showed no correlation with GA.

Diagnostic performance of serum $h C G$, progesterone, and selected miRNAs, and in combination

The diagnostic performance of the different serum markers for detecting EP was analyzed by ROC curve analysis in a cohort of 34 EP patients and 66 non-EP controls (Table 3, Fig. 3A). The sensitivities at the predefined specificities of $90 \%$ and $95 \%$ and the specificities at the predefined sensitivities of $90 \%$ and $95 \%$ were calculated for all markers 
Table 2. Patient age, GA, and serum concentrations of hCG, progesterone, and miRNAs in women with EP, SA,or VIP. a Data are expressed as the median (interquartile range). b Dunn multiple-comparison post hoc test. ${ }^{\mathrm{C} S}$, not statistically significant $(\mathrm{P} \geq 0.05)$. ${ }^{\mathrm{d}}$ miRNA concentrations are expressed relative to $18 \mathrm{~S}$ rRNA

\begin{tabular}{|c|c|c|c|c|c|c|c|}
\hline & $\operatorname{VIP}(n=36)^{a}$ & $\operatorname{EP}(n=34)^{a}$ & SA $(n=30)^{a}$ & $\begin{array}{c}\text { Kruskal- } \\
\text { Wallis } \\
\text { test }\end{array}$ & EP vs $S^{b}$ & EPvs VIPb & SA vs VIPb \\
\hline Patient age, years & $28(25-31.5)$ & $30(27-34)$ & $28.5(25-33)$ & $\mathrm{NS}^{\mathrm{c}}$ & NS & NS & NS \\
\hline GA, weeks & $7.79(5.75-8.57)$ & $6.86(5.97-7.97)$ & $7.00(6.12-9.12)$ & NS & NS & NS & NS \\
\hline hCG, IU/L & 56695 (10161-85715) & 1550 (489.9-4977) & 3148 (1007-4913) & $<0.001$ & NS & $\mathrm{p}<0.001$ & $\mathrm{p}<0.001$ \\
\hline Progesterone,ng/ml & $28.55(22.36-34.95)$ & $7.85(5.46-11.43)$ & $12.17(5.95-16.85)$ & $<0.001$ & NS & $\mathrm{p}<0.001$ & $\mathrm{p}<0.001$ \\
\hline $\operatorname{miR}-218^{d}$ & $0.95(0.23-2.83)$ & $1.25(0.28-3.34)$ & $0.16(0.064-1.06)$ & $<0.01$ & $\mathrm{p}<0.05$ & NS & $\mathrm{p}<0.05$ \\
\hline $\operatorname{miR}-223^{d}$ & $0.33(0.14-0.45)$ & $0.44(0.19-0.72)$ & $1.64(0.65-2.62)$ & $<0.001$ & $\mathrm{p}<0.001$ & NS & $\mathrm{p}<0.001$ \\
\hline $\operatorname{miR}-873^{d}$ & $5.10(2.65-6.43)$ & $0.089(0.031-2.28)$ & $3.79(2.14-6.07)$ & $<0.001$ & $\mathrm{p}<0.001$ & $\mathrm{p}<0.001$ & NS \\
\hline $\operatorname{miR}-141^{d}$ & $0.090(0.027-0.26)$ & $0.68[0.25-1.05]$ & $0.49[0.17-1.63]$ & $<0.001$ & NS & $p<0.01$ & $p<0.001$ \\
\hline MiR-323d & $0.61(0.11-2.52)$ & $2.45(1.16-2.02)$ & $0.47(0.097-2.45)$ & $<0.001$ & $\mathrm{p}<0.01$ & $p<0.01$ & NS \\
\hline
\end{tabular}

Fig. 2. Expression of hCG, progesterone and five miRNAs in different weeks of gestational age (GA). (A-G) Serum concentrations of hCG, progesterone, miR218, miR-233, miR-141, miR-873, and miR-323 according to GA at the time of emergency department visit in patients with EP, SA, and VIP.

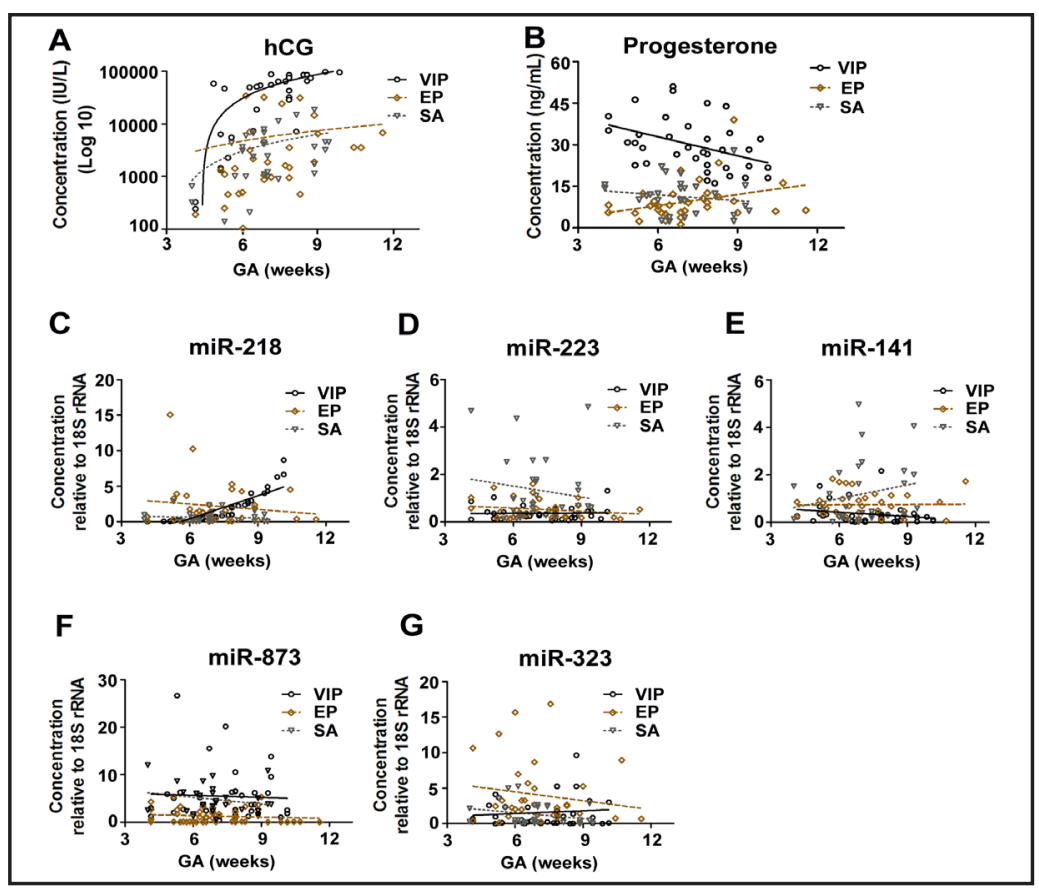

individually and in combination as shown in Table 3. As single markers, the AUC values were as follows: progesterone vs. hCG: 0.82 [95\% confidence interval (CI) 0.73-0.91] vs. 0.74 (95\% CI 0.65-0.84); miR-323 and miR-873: 0.74 (95\% CI 0.64-0.84) and 0.84 (95\% CI 0.760.92), respectively. MiR-323 had a higher sensitivity for the detection of EP than hCG and progesterone at the fixed specificities of $90 \%$ and $95 \%$ ( $47.60 \%$ and $32.35 \%$, respectively). However, miR-873 had the highest sensitivity for the detection of EP as a single marker at the fixed specificities of $90 \%$ and $95 \%$ (61.76\% and 55.88\%, respectively). To determine the value of the miRNAs as biomarkers, we conducted a multivariate logistic regression analysis to compare the different combinations of biomarkers with that of the dual markers of hCG plus progesterone as a standard reference. The AUCs of different multimarker combinations were 0.84 to 0.92 (Table 3, Fig. 3B). The combinations of hCG, progesterone and miR-323 produced higher sensitivities at $67.65 \%$ and $50.00 \%$ than those of hCG plus progesterone at $55.85 \%$ and $44.12 \%$ with specificities of $90 \%$ and $95 \%$, respectively (both $P<0.05$ ). The combinations of hCG, progesterone and miR-873 produced the highest sensitivities of $79.41 \%$ and $64.71 \%$ compared with $55.85 \%$ and $44.12 \%$ for hCG plus progesterone with specificities of $90 \%$ and $95 \%$, respectively (both $P<0.01$,). The combinations of $\mathrm{hCG}$, progesterone, miR873 and miR-323 produced higher sensitivities at $70.59 \%$ and $52.94 \%$ than $55.88 \%$ and 
Lu et al.: miR-873 is a Potential Marker in Ectopic Pregnancy

Table 3. Diagnostic accuracies of measurements of single markers and of multimarker combinations for predicting EP in patients with abdominal pain and/or vaginal bleeding at predefined specificities and sensitivities. ${ }^{a} \mathrm{AUC}$, area under the curve. ${ }^{\mathrm{b}}$ sensitivities, and specificities of each multimarker combination were compared with those of $\mathrm{hCG}+$ progesterone in the same column. ${ }^{\mathrm{C}} \mathrm{P}<0.05,{ }^{\mathrm{d}} \mathrm{P}<0.01$; ${ }^{\mathrm{e}} \mathrm{P}<0.05, \mathrm{hCG}+$ progesterone+miR-873 compare to hCG+progesterone+miR-323 or hCG+progesterone+miR-873+miR-323

\begin{tabular}{|c|c|c|c|c|c|}
\hline & \multirow[b]{2}{*}{$\operatorname{AUC}(95 \% \mathrm{CI})$} & \multicolumn{2}{|c|}{ Sensitivity at: } & \multicolumn{2}{|c|}{ Specificity at: } \\
\hline & & $90 \%$ Specificity, $\%$ & $95 \%$ Specificity, $\%$ & $90 \%$ Sensitivity, $\%$ & $\begin{array}{c}95 \% \\
\text { Sensitivity, \% }\end{array}$ \\
\hline hCG & $0.74(0.65-0.84)$ & 47.06 & 41.48 & 39.39 & 36.36 \\
\hline Progesterone & $0.82(0.73-0.91)$ & 41.48 & 35.29 & 59.09 & 43.94 \\
\hline miR-218 & $0.39(0.27-0.51)$ & 17.65 & 5.88 & 31.82 & 3.03 \\
\hline miR-223 & $0.58(0.47-0.69)$ & 0 & 0 & 28.27 & 24.24 \\
\hline miR-141 & $0.38(0.27-0.49)$ & 11.76 & 0 & 34.85 & 33.33 \\
\hline miR-873 & $0.84(0.76-0.92)$ & 61.76 & 55.88 & 59.09 & 50.00 \\
\hline miR-323 & $0.74(0.64-0.84)$ & 47.06 & 32.35 & 43.94 & 34.85 \\
\hline $\mathrm{hCG}+$ progesterone ${ }^{\mathrm{b}}$ & $0.84(0.75-0.92)$ & 55.88 & 44.12 & 59.09 & 48.48 \\
\hline $\mathrm{hCG}+$ progesterone+miR-323 & $0.87(0.79-0.94)$ & $67.65^{c}$ & $50.00^{c}$ & 63.64 & 56.06 \\
\hline $\mathrm{hCG}+$ progesterone+miR-873 & $0.91(0.86-0.97)$ & $79.41^{\mathrm{d}, \mathrm{e}}$ & $64.71^{\mathrm{d}, \mathrm{e}}$ & $84.85^{\mathrm{d}, \mathrm{e}}$ & $80.82^{\mathrm{d}, \mathrm{e}}$ \\
\hline hCG + progesterone+miR-873+miR-323 & $0.92(0.87-0.98)$ & $70.59^{\mathrm{d}}$ & $52.94^{\mathrm{c}}$ & 66.67 & 50.00 \\
\hline
\end{tabular}

Fig. 3. Detection of single markers and multiple makers in EP and non-EP patients. (A) ROC curve analysis of hCG, progesterone, miR-218, miR-233, miR-141, miR-873, and miR-323 as single markers in the validation set of 34 patients with EP and 66 non-EP controls. (B) ROC curve analysis of hCG and progesterone; hCG, progesterone, and miR873; hCG, progesterone, and miR-323; and hCG, progesterone, miR-873, and miR-323 as multiple markers in the validation set of 34 patients with EP and 66 non-EP controls, respectively.

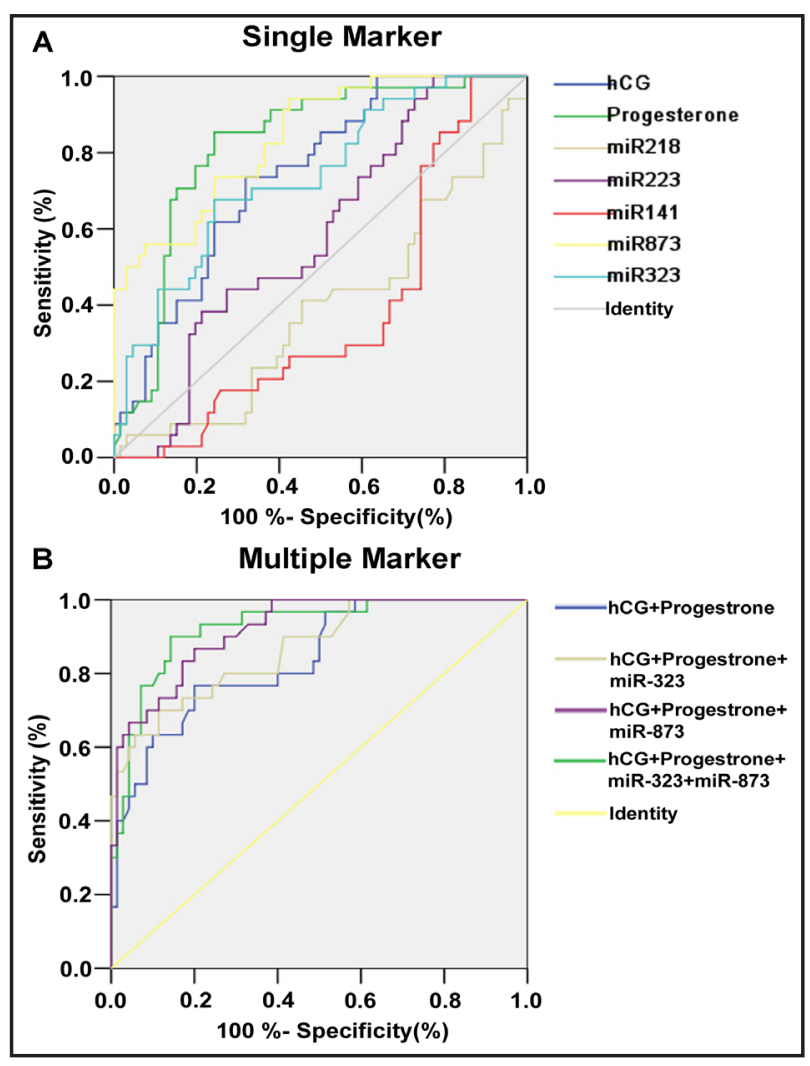

$44.12 \%$ for hCG plus progesterone at specificities of $90 \%$ and $95 \%$, respectively $(P<0.01$ and $P<0.05$, respectively). Moreover, the combination of hCG, progesterone and miR-873 produced higher sensitivities than the combinations of hCG, progesterone, and miR-323 and the combinations of hCG, progesterone, miR-323, and miR-873 (both $P<0.05$. at specificities of $90 \%$ and $95 \%$, respectively).

\section{Discussion}

Approximately $15 \%$ of pregnancies are associated with complications such as preeclampsia, EP, gestational diabetes mellitus, spontaneous abortion, and preterm delivery among others [2,16-18]. EP is an important cause of maternal morbidity and can be fatal when 
undetected because of the potential for tubal rupture and hemorrhage[19]. The diagnosis of EP currently relies on a combination of sonographic findings and serum hCG measurements; however, EP is difficult to diagnose at an early stage because approximately one third of all cases have no clinical signs and $9 \%$ of patients have no symptoms prior to rupture[20]. In addition, single hCG measurements cannot distinguish between EP, SA, and VIP, requiring repeated hCG measurements that are not only time consuming and costly, but are also associated with a risk of rupture between measurements [19]. Therefore, the identification of accurate serum biomarkers for the early diagnosis of EP would be of great clinical value. However, research needs to be carried out in women, as there are no suitable animal models of tubal ectopic pregnancy, and serum concentrations of putative biomarkers are measured retrospectively in women who have been diagnosed with EP, VIP, and SA, which is associated with problems in the interpretation of biomarker utility[19]. Serum concentrations of these biomarkers vary with GA, and although some markers can distinguish between EP and VIP, they cannot discriminate between EP and SA [19]. Nevertheless, many markers have been tested for their capacity to detect EP at an early stage. Serum biomarkers of EP include those indicative of abnormal embryo/trophoblast growth (hCG, pregnancy-associated plasma protein-A, and activin-A among others) abnormal corpus luteum function (progesterone, estradiol), markers of growing pregnancy in the Fallopian tube, markers of inflammation and peritoneal irritation, and uterine markers of normal implantation, which would be disrupted in the presence of tubal implantation. Among these, hCG is the only biomarker routinely used in the clinic, and progesterone is sometimes added to the routine hCG/ultrasound diagnostic protocol despite controversies regarding its accuracy[19]. Activin A, which is not used in clinical practice, is a potentially important biomarker that is currently being investigated $[21,22]$.

miRNAs have shown potential as biomarkers for the detection of pregnancy-related diseases. The value of miRNAs lies not only in their noninvasive nature, but also in the fact that they are stable and their concentrations do not vary throughout pregnancy[16]. In 2008, Chim et al. compared the profiles of 157 miRNAs between third-trimester placental tissues and the corresponding maternal blood cells and identified 34 miRNAs that were present at more than ten-fold higher concentrations in maternal blood cells [23]. Of these, four miRNA (miRs$141,149,299-5 p$, and 135b) were confirmed to be of placental origin. Subsequent studies identified many placental-specific miRNAs, confirming that these molecules are released into the maternal circulation and could serve as serum biomarkers. To detect miRNAs with diagnostic potential for pregnancy-associated complications, researchers have compared the serum concentrations of miRNAs between patients with specific disorders and healthy controls, which resulted in the identification of a large number of differentially expressed miRNAs, many of which were confirmed as promising biomarkers for specific disorders. Zhao et al. examined the diagnostic accuracy of miRNAs for the detection of EP and showed that miR-517a, miR-519d, and miR-525-3p were significantly lower, whereas miR-323-3p was significantly higher in EP and SA than in VIP[8]. Furthermore, the combined assessment of miR-323-3p, hCG, and progesterone showed a sensitivity of $77.8 \%$ for the detection of EP at a fixed specificity of $90 \%$. In the present study, we measured the concentration of 21 pregnancy-associated miRNAs as well as hCG and progesterone in serum samples from patients with EP, VIP, and SA. Of five miRNAs differentially expressed between these patients, miR-873 and miR-223 were significantly downregulated in EP compared with SA and did not change significantly according to GA. MiR-323 was also significantly higher in EP than in VIP and SA, similar to a previously study[8], and the combined assessment of miR-323$3 p$, hCG, and progesterone showed a sensitivity of $67.65 \%$ for the detection of EP at a fixed specificity of $90 \%$, and in combination with other markers showed that the combination of miR-323 with hCG and progesterone and miR-873 had the highest AUC for the prediction of EP. As a single marker, miR-873 had the highest sensitivity at $61.76 \%$ (at a fixed specificity of $90 \%$ ), suggesting its potential as a biomarker for the early detection of EP. And combination with hCG, and progesterone showed a sensitivity of $79.41 \%$ for the detection of EP at a fixed specificity of $90 \%$. However, combination with hCG, progesterone, miR-873 and miR-323

\section{KARGER}




\section{Cellular Physiology Cell Physiol Biochem 2017;41:2513-2522 \begin{tabular}{c|c} 
DOI: 10.1159/000475946 & O 2017 The Author(s). Published by S. Karger AG, Basel \\
www.karger.com/cpb
\end{tabular}}

Lu et al.: miR-873 is a Potential Marker in Ectopic Pregnancy

showed a sensitivity of $70.59 \%$ for the detection of EP at a fixed specificity of $90 \%$.

miR-873 was previously shown to be among five miRNAs downregulated in EP in a miRNA microarray comparison of pregnancy derived tissues from patients with EP and those with voluntary termination of pregnancy (VTOP) [24], which is consistent with the results of the present study. However, Dominguez et al. showed that miR-223 was upregulated in EP, which differs from our results showing that miR-223 expression was significantly lower in EP than in SA. In the study by Dominguez et al. the upregulation of miR-223 was further validated by real-time PCR in a larger sample of embryonic tissues from EP and VTOP, and it was further shown to target GALNT1, a gene in the mucin biosynthesis pathway. Nevertheless, the study by Dominguez et al. did not compare EP and SA [24]; therefore, whether the results of the two studies are in accordance is difficult to determine. Zhi et al. showed that miR-223 was shown to inhibit bladder cancer cell migration and invasion by downregulating its target ZEB1 [25]. In addition, miR-223 was shown to be a potential target for the treatment of ischemic arrhythmias, as miR-223 repressed the expression of the voltage-gated $\mathrm{K}+$ channel Kv4.2 [26]. miR-223 suppresses cardiomyocyte hypertrophy by modulating the expression of its target TNNI3K, a troponin interacting kinase [27]. In a study identifying differentially expressed miRNAs in polycystic ovarian syndrome, miR-223 was found to be downregulated and associated with increased risk of ovarian hyperstimulation syndrome [28]. Exosomes mediates miR-223 could inhibit Platelet-Derived Growth Factor Receptor-Beta (PDGFR $\beta$ ) expression in Vascular Smooth Muscle cells [29]. miR-323 was shown to upregulate VEGF-A and increase vascularization in prostate cancer [30]. In a recent study, miR-873 was shown to be downregulated in breast tumors and to play a tumor suppressor role by targeting cyclindependent kinase 3, leading to the inhibition of ER- $\alpha$ activity and the suppression of breast cancer cell proliferation and tumor growth [31]. Insulin-like growth factor 2 mRNA binding protein 1 (IGFBP1) was identified as a target of miR-873 in glioblastoma [32]. IGFBP1, which is produced at high levels in the human endometrium, has been suggested as a marker of EP $[33,34]$. IGF-I expression in the endometrium is induced by estrogen and mediates estrogen action, and IGF-II expression is associated with endometrial differentiation. IFGBP1 regulates the function of IGF at the embryo-endometrial interface. The modulation of IGFBP1 by miR873 and their potential role in EP therefore merits further investigation.

In conclusion, we screened potential miRNA biomarkers for the detection of EP in an ED setting by analyzing a population of miRNAs with a known relationship to pregnancy or placental expression and by comparing their levels between patients with EP, VIP and SA. We identified five differentially expressed miRNAs and showed that miR-873 had the highest specificity as a single marker and is therefore a promising candidate biomarker for the early detection of EP. Further studies with a larger cohort are necessary to validate our results. Furthermore, assessment of other markers such as hCG and progesterone in combination with miRNAs may help improve the diagnostic performance of the identified miRNAs as biomarkers and should be performed in future studies.

\section{Acknowledgments}

This work was funded by the Medical Guiding Project of the Science and Technology Commission of Shanghai Municipality (15411961100 and 16411963000); HundredTalent Program of Shanghai Jiaotong University (XBR2013097); Key Project of Shanghai Municipal Bureau of health (20124027); Appropriate technology of Municipal Hospital in Shanghai (SHDC12014208); Emerging frontier project of Municipal Hospital in Shanghai (SHDC12016111), and General project of Jinshan distract of Shanghai Municipal Commission of Health and Family planning (JSKJ-KTMS-2016-02).

\section{Disclosure Statement}

The authors declare that they have no conflicts of interest with the contents of this article. 


\section{Cellular Physiology Cell Physiol Biochem 2017;41:2513-2522 \begin{tabular}{l|l} 
and Biochemistry & $\begin{array}{l}\text { DOI: 10.1159/000475946 } \\
\text { Published } 2017 \text { (o) } 2017 \text { The Author(s). Published by S. Karger AG, Basel } \\
\text { www.karger.com/cpb }\end{array}$
\end{tabular}}

Lu et al.: miR-873 is a Potential Marker in Ectopic Pregnancy

\section{References}

1 Ayaz A, Emam S, Farooq MU: Clinical course of ectopic pregnancy: A single-center experience. J Hum Reprod Sci 2013;6:70-73.

2 Murray H, Baakdah H, Bardell T, Tulandi T: Diagnosis and treatment of ectopic pregnancy. CMAJ 2005;173:905-912.

3 Farquhar CM: Ectopic pregnancy. Lancet 2005;366:583-591.

4 Shaunik A, Kulp J, Appleby DH, Sammel MD, Barnhart KT: Utility of dilation and curettage in the diagnosis of pregnancy of unknown location. Am J Obstet Gynecol 2011;204:130 e131-136.

$>5$ Sowter MC, Farquhar CM: Ectopic pregnancy: An update. Curr Opin Obstet Gynecol 2004;16:289-293. Barnhart K, Sammel MD, Chung K, Zhou L, Hummel AC, Guo W: Decline of serum human chorionic gonadotropin and spontaneous complete abortion: Defining the normal curve. Obstet Gynecol 2004;104:975-981.

7 Kadar N, Caldwell BV, Romero R: A method of screening for ectopic pregnancy and its indications. Obstet Gynecol 1981;58:162-166.

8 Zhao Z, Zhao Q, Warrick J, Lockwood CM, Woodworth A, Moley KH, Gronowski AM: Circulating microrna mir-323-3p as a biomarker of ectopic pregnancy. Clin Chem 2012;58:896-905.

-9 Warrick J, Gronowski A, Moffett C, Zhao Q, Bishop E, Woodworth A: Serum activin A does not predict ectopic pregnancy as a single measurement test, alone or as part of a multi-marker panel including progesterone and hCG. Clin Chim Acta 2012;413:707-711.

10 Bartel DP: Micrornas: Genomics, biogenesis, mechanism, and function. Cell 2004;116:281-297.

-11 Mitchell PS, Parkin RK, Kroh EM, Fritz BR, Wyman SK, Pogosova-Agadjanyan EL, Peterson A, Noteboom J, O'Briant KC, Allen A, Lin DW, Urban N, Drescher CW, Knudsen BS, Stirewalt DL, Gentleman R, Vessella RL, Nelson PS, Martin DB, Tewari M: Circulating micrornas as stable blood-based markers for cancer detection. Proc Natl Acad Sci U S A 2008;105:10513-10518.

$\checkmark 12$ Kotlabova K, Doucha J, Hromadnikova I: Placental-specific microrna in maternal circulation--identification of appropriate pregnancy-associated micrornas with diagnostic potential. J Reprod Immunol 2011;89:185191.

13 Miura K, Miura S, Yamasaki K, Higashijima A, Kinoshita A, Yoshiura K, Masuzaki H: Identification of pregnancy-associated micrornas in maternal plasma. Clin Chem 2010;56:1767-1771.

14 Mouillet JF, Chu T, Hubel CA, Nelson DM, Parks WT, Sadovsky Y: The levels of hypoxia-regulated micrornas in plasma of pregnant women with fetal growth restriction. Placenta 2010;31:781-784.

15 Zhang Y, Diao Z, Su L, Sun H, Li R, Cui H, Hu Y: Microrna-155 contributes to preeclampsia by downregulating cyr61. Am J Obstet Gynecol 2010;202:466.e461-467.

16 Zhao Z, Moley KH, Gronowski AM: Diagnostic potential for mirnas as biomarkers for pregnancy-specific diseases. Clin Biochem 2013;46:953-960.

17 Wallis AB, Saftlas AF, Hsia J, Atrash HK: Secular trends in the rates of preeclampsia, eclampsia, and gestational hypertension, United States, 1987-2004. Am J Hypertens 2008;21:521-526.

18 Hunt KJ, Schuller KL: The increasing prevalence of diabetes in pregnancy. Obstet Gynecol Clin North Am 2007;34:173-199.

19 Cartwright J, Duncan WC, Critchley HO, Horne AW: Serum biomarkers of tubal ectopic pregnancy: Current candidates and future possibilities. Reproduction 2009;138:9-22.

20 Tay JI, Moore J, Walker JJ: Ectopic pregnancy. BMJ 2000;320:916-919.

-21 Roghaei MA, Sabet F, Mohamadi K: Diagnostic accuracy of serum activin a in detection of ectopic pregnancy. J Res Med Sci 2012;17:378-381.

22 Refaat B: Role of activins in embryo implantation and diagnosis of ectopic pregnancy: A review. Reprod Biol Endocrinol 2014; 25:112-116.

23 Chim SS, Shing TK, Hung EC, Leung TY, Lau TK, Chiu RW, Lo YM: Detection and characterization of placental micrornas in maternal plasma. Clin Chem 2008;54:482-490.

24 Dominguez F, Moreno-Moya JM, Lozoya T, Romero A, Martinez S, Monterde M, Gurrea M, Ferri B, Nunez MJ, Simon C, Pellicer A: Embryonic mirna profiles of normal and ectopic pregnancies. PLoS One 2014;9:e102185.

25 Zhi Y, Pan J, Shen W, He P, Zheng J, Zhou X, Lu G, Chen Z, Zhou Z: Ginkgolide b inhibits human bladder cancer cell migration and invasion through microrna-223-3p. Cell Physiol Biochem 2016;39:1787-1794. 


\section{Cellular Physiology Cell Physiol Biochem 2017;41:2513-2522 and Biochemistry \begin{tabular}{l|l} 
DOI: 10.1159/000475946 \\
Published online: May 04, 2017
\end{tabular} \begin{tabular}{l}
$\begin{array}{l}\text { O 2017 The Author(s). Published by S. Karger AG, Basel } \\
\text { www.karger.com/cpb }\end{array}$ \\
\hline
\end{tabular} \\ Lu et al.: miR-873 is a Potential Marker in Ectopic Pregnancy}

-26 Liu X, Zhang Y, Du W, Liang H, He H, Zhang L, Pan Z, Li X, Xu C, Zhou Y, Wang L, Qian M, Liu T, Yin H, Lu Y, Yang B, Shan H: Mir-223-3p as a novel microrna regulator of expression of voltage-gated k+ channel kv4.2 in acute myocardial infarction. Cell Physiol Biochem 2016;39:102-114.

-27 Wang YS, Zhou J, Hong K, Cheng XS, Li YG: Microrna-223 displays a protective role against cardiomyocyte hypertrophy by targeting cardiac troponin i-interacting kinase. Cell Physiol Biochem 2015;35:1546-1556.

28 Zhao C, Liu X, Shi Z, Zhang J, Jia X, Ling X: Role of serum mirnas in the prediction of ovarian hyperstimulation syndrome in polycystic ovarian syndrome patients. Cell Physiol Biochem 2015;35:10861094.

-29 Tan M, Yan HB, Li JN, Li WK, Fu YY, Chen W, Zhou Z: Thrombin stimulated platelet-derived exosomes inhibit platelet-derived growth factor receptor-beta expression in vascular smooth muscle cells. Cell Physiol Biochem 2016;38:2348-2365.

30 Gao Q Yao X, Zheng J: Mir-323 inhibits prostate cancer vascularization through adiponectin receptor. Cell Physiol Biochem 2015;36:1491-1498.

-31 Cui J, Yang Y, Li H, Leng Y, Qian K, Huang Q, Zhang C, Lu Z, Chen J, Sun T, Wu R, Sun Y, Song H, Wei X, Jing P, Yang X, Zhang C: Mir-873 regulates eralpha transcriptional activity and tamoxifen resistance via targeting cdk3 in breast cancer cells. Oncogene 2015;34:4018.

-32 Wang RJ, Li JW, Bao BH, Wu HC, Du ZH, Su JL, Zhang MH, Liang HQ: Microrna-873 (mirna-873) inhibits glioblastoma tumorigenesis and metastasis by suppressing the expression of igf $2 \mathrm{bp} 1$. J Biol Chem 2015;290:8938-8948.

-33 Rutanen EM: Insulin-like growth factors in endometrial function. Gynecol Endocrinol 1998;12:399-406.

-34 Rutanen EM: Insulin-like growth factors and insulin-like growth factor binding proteins in the endometrium. Effect of intrauterine levonorgestrel delivery. Hum Reprod 2000;15:173-181. 